\title{
A Binding Rule for Government-binding Parsing
}

\author{
Nelson CORREA \\ IIBM Thomas J. Watson Rescarch Center \\ P. O. Box 704 \\ Yorktown Heights, NY 10598 \\ USA
}

\begin{abstract}
In this paper I propose a Binding rule for the identification of pronoun and anaphor referents in phrase-structure trees, assuming the general framework of the Government-binding theory outlined by Chomsky (1981). The Binding rule, specified by means of an attribute grammar, is a particular instantiation of the Free Indexing rule and binding axioms in Chomsky's Binding theory, with certain empirical and practical advantages. The complexities of the Binding rule proposed, as well as that inherent in Chomsky's Binding theory, are studied, and it is shown that the new rule is more psychologically plausible and computationally efficient than the original theory on which it is based. The fragment of the attribute grammar shown here is part of an English grammar and parser being developed in the Prolog and PLNLP languages.
\end{abstract}

\section{Introduction}

Binding is a component subtheory of Governmentbinding which applies in the derivation of the logical form of utterances from their surface rcpresentation. The area of semantic interpretation dealt with by the binding theory is that of anaphora. Binding theory defines only syntactic conditions on anaphora; the reader is referred to /Hobbs, 1978/ for some of the extra-syntactic factors that might be involved. Binding assumes an Indexing rule which applies to an input S-Structure tree and annotates it, assignitig to every NP' node in the input tree a referential index, which represents the coreference relation of the NP' with other NPs in the input.

In this paper research is continued on the use of attribute grammars to provide a fully explicit and computationally oriented statement of the Government-binding (GB) theory /Correa, 1987/.
The Binding rule presented here improves over the standard statement of the Binding theory in two respects: From an empirical point of view, the new rule accounts for crossover binding phenomena /Kuno, 1987/ without recourse to reconstruction /Chomsky, 1981/; from a practical point of view, the new rule is more computationally sensible than the generate-and-test approach understood in Chomsky's theory, and hence is a plausible candidate for incorporation in natural-language parsers that account for anaphora. I'revious literature on Gl3 parsing/Wehrli, 1984; Sharp, 1985; Kashket, 1986; Kuhns, 1986; Abncy, 1986/ has not addressed the issue of implementation of the Binding theory. 'The present paper intends in part to fill this gap.

In the development below I will assume that the reader is familiar with attribute grammars and the basic concepts and terminology of Governmentbinding, although not necessarily with the Binding theory. The reader is referred to Waite and Goos (1984) for a concise introduction to attribute grammars, and Sells (1985) for the basic assumptions of Government-binding.

\section{Chomsky's Binding Theory}

Binding theory defines the syntactic constraints on coreference that exist between the noun phrases in a sentence. In the course of doing this, the theory indirectly determines constraints on the distribution of eertain kinds of noun phrases. In this section we review the standard formulation of the Binding theory; the reader already familiar with it may proceed to the next section.

The referential possibilities of a noun phrase depend on the functional type of the $\mathrm{NP}$ and the Binding conditions for that type. Governmentbinding distinguishes three types of overt NP, shown in (1).

1 Sharp (1985) checks correctriess of binding in traces; we consider lexical NPs here. 
(1) a. anaphor (reflexive and reciprocal)

b. pronominal

c. referential

$\Delta \mathrm{n}$ anaphor is an expression that has no independent reference and must take its reference from some other expression in the sentence in which it occurs. English has reflexive and reciprocal anaphors, such as 'themselves' and 'each other' in (2). The NP' from which an anaphor or pronominal takes its reference is called its antecedent. since an anaphor must have an antecedent within the sentence in which it is used, we obtain the contrast between (2.a) and (2.b). If there is no appropriate antecedent, the string is ill-formed at the Logical Form level. The antecedent of the anaphor must, furthermore, c-command the anaphor and be found within a certain local domain, notions to be made precise below. Thus, in (2.c), although there is a potential antecedent for the anaphor, namely 'Greeks', it is not within the required local domain. In (2.d), there is a potential antecedent 'donkey', but it does not c-command the anaphor. Hence tie string is also ill-formed.

(2) a. Greeks like themselves/ each other.

b. * Each other/ Themselves like Greeks.

c. * Greeks $s_{\mathrm{i}}$ think that each other / $_{\mathrm{i}}$ themselves $\mathrm{i}_{\mathrm{i}}$ are smart.

d. * Every man who owns a donkey beats $_{\mathrm{i}}$ itself.

A pronominal is a pronoun in any of its inflected forms (e.g., as due to agreement and Casemarking), as in (3). Pronominals exhibit a distribution in phrase structure trees nearly complementary to that of anaphors. A pronominal need not pick its reference from some other NP in the sentence, but rather may have independent (deictic) interpretation, as in the first reading of (3.a). The pronominal may also be read anaphorically, having its reference determined by some other NP in the sentence (3.a-b). In this case, though, the antecedent must either be outside the local domain of the pronominal, or not c-command it. Hence, the assigned coreference in (3.a-b) is possible, while that in (3.c) is not. Within a local domain, where an anaphor must have an antecedent, a pronominal cannot.

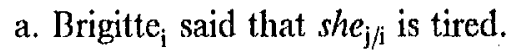
b. Every man who owns a donkey ${ }_{i}$ beats $i t_{\mathrm{i}}$.
c. * Sibylle $\mathrm{i}_{\mathrm{i}}$ loves her .

Lexical or fully referential expressions are names like 'John' and 'the man' in (4); the class includes all nominals headed by a common or proper noun. A referential expression defines its reference independently and must be free in every domain, in the sense that it may not have a c-commanding antecedent. Thus the interpretations in (4.a-b) are unwarranted. Coreference between referential NP's is possible only if the first NP does not c-command the second (4.c-d); the result, though, may be awkward or place emphasis on the anaphoric noun phrase.
a. John $_{\mathrm{i}}$ likes John $n_{\mathrm{i}}$.
b. *John $n_{\mathrm{i}}$ wants that $J o h n_{\mathrm{i}}$ leaves.
c. The man who hired John $n_{\mathrm{i}}$ likes $J o h n_{\mathrm{i}}$.
d. John $n_{\mathrm{i}}$ came and $J o h n_{\mathrm{i}}$ left.

The most difficult area of the Binding theory is the formulation of the notion local domain referred to above. This notion is defined such that it is identical for anaphors and pronouns. We notc in advance, however, that while the notion is nearly identical for both, it should not be defined the same, as sentences (5.a-b) show (Chomsky, 1986). In this paper we shall not be concerned with the solution of this still open problem.

$$
\begin{aligned}
& \text { (5) a. The children like each other's } s_{\mathrm{i}} \text { pictures. } \\
& \text { b. The children }{ }_{\mathrm{i}} \text { like their }{ }_{\mathrm{j}} \text { pictures. }
\end{aligned}
$$

Chomsky's axiomatic statement of the Binding theory is as follows. Chomsky (1981) assumes a Free Indexing rule which applics at LF and assigns (randomly) a referential index to every $\mathrm{NP}^{\prime}$ in the input structure. Two NPs are said to be coreferential if they bear the same referential index. The indexing rule massively overgenerates logical forms, and indiscriminately assigns unwarranted coreference relations. The annotated structures produced by the rule are subject to a number of well-formedness conditions, which are constraints on the assigned coreference relations.

The most elementary condition is the agreement condition (6). The main component of the theory is given by the Binding axioms (7), where the notions of binding and local domain are as in (8) and (9), respectively. Notice that the definition (9) of local domain does not distinguish between anaphors and pronominals, and thus is problematic, as the examples (5) indicate. We assume this definition, though, for the development below. The notion of c-command used in (8) is given in (10), 
(6)

\section{Agreement Condition}

If $\mathrm{NP}_{1}$ and $\mathrm{NP}_{2}$ are coindexed, then their agreement features $\Lambda G R=<$ Person, Gender, Number > agree.

\section{Biuding Axioms}

A. An anaphor must be bound within its local domain.

B. A pronominal must be free within its local domain.

C. A referential expression must be free in every domain.

(8) For nodes $\alpha$ and $\beta, \alpha$ binds $\beta$ if (i) $\alpha$ is coindexed with $\beta$, and (ii) $\alpha c$-commands $\beta$. $\Lambda$ node $\alpha$ is free (within a given domain) if it is not bound (within that domain).

(9) The local domain of a node $\alpha$ is the subtree dominated by $\operatorname{MGC}(\alpha)$, where

$\operatorname{MGC}(\alpha)$, the minimal governing category of $\alpha$, denotes the maximal projection $\mu$ nearest to $x$ such that

$$
\begin{aligned}
& \mu \text { dominates } \alpha, \text { and } \\
& \mu \text { has an accessible Subject, and } \\
& \mu \text { dominates a governor } \gamma \text { of } \alpha
\end{aligned}
$$

(10) For nodes $\alpha$ and $\beta, \alpha$ c-commands $\beta$ if the first branching node dominating $\alpha$ also dominates $\beta$.

It is a straightforward task to verify that the Binding axioms in (7) explain the grammaticality judgements and interpretation possibilities of the examples presented thus far, except those in (5). The theory is explanatorily adequate, in the sense that it applies to a wide range of natural languages.

\section{Procedural Binding}

The Binding theory just outlined follows the style of most recent work within the Governmentbinding framework. Extremely general rules, such as the Free Indexing rule, are assumed for the generation and annotation of syntactic structure; the bulk of the grammar then consists of wellformedness conditions or axioms that must be satisfied by the generated structures. This approach; due to its extreme inefficiency, is problematic as a model of linguistic performance or natural language parsing. It secms more appropriate to view the general rules and axioms that constrain them as high-level specifications of certain grammatical processes, rather than as models of how the processes are actually carried out.

The refinement of the general rules and axioms associated with them into procedural rules which may be used to derive structure that already satisfies the axioms is not a straightforward task, and has only recently begun to be addressed / $\Lambda$ bney and Cole, 1986; Barton, 1984/. The incorporation of axioms into the rules leads to grammars which are more sensitive to psychological issues/linguistic processing, rather than mere linguistic description. It seems clear that only these new rules may be used in practical natural language parsers. Furthermore, the formulation of procedural mechanisms provides a new way of looking at linguistic phenomena, which may in turn lead to insights for the solution of outstanding problems. I offer the following Binding rule as an illustration.

The Binding rule is defined by means of attribution rules associated with productions in the base. It applies at S-Structure and assigns to each NP' node in the structure a referential index, in such way that the Binding axioms are satisfied by the assignment. The generate-and-test method implicit in Chomsky's account is avoided. In those S-Structures for which there is no possible correct assignment, the rule blocks, and the structures are marked ill-formed, due to some violation of the Binding theory. The rule applies after the functional type of every NP has been determined, according to lexical features of the head nominal and principles of the Government and Case theories. Functional classification of an NP consists of determining the values of its attributes anaphoric and pronominal /van Riemsdijk and Williams, 1986/. The first approximation to the rule is limited to cases of backward reference only; assignment of forward coreference, as in (11), will not be covered by the rule. Also, we ignore cases where referential expressions may be used anaphorically, as in (4.c-d).

(11) Men who met her ${ }_{i}$ saw how kind Mary $y_{i}$ was.

The formulation of the rule relies crucially on the following hypothesis: For every NP node in an S-Structure, it is possible to define two sets of nominal expressions $A A S$ and $P A S$, which contain, respectivcly, potential anaphoric and pronominal antecedents. Given a mechanism to compute the two sets noted, an antecedent for the current node may be selected from the appropriate set, according to the current node's functional type, as in (12). Attribution rule (12) is associated with every production for NP and defines the value of the NP's referential index. The function select-from takes an ordered set as argument and selects (arbitrarily) the 
first element that morphologically agrees with the NP. ${ }^{2}$

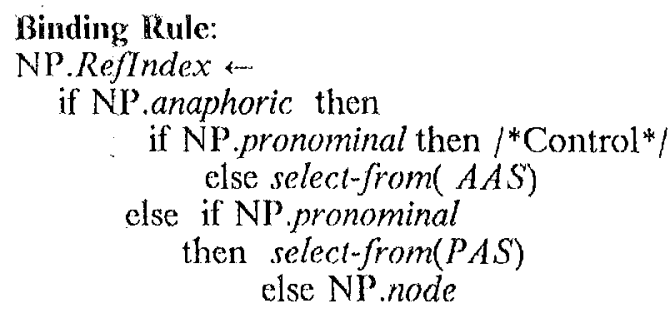

The main component of the Binding rule consists of the attribution rules that define the values of the $A A S$ and $P A S$ sets at each node. I now proceed to describe the types of the attributes involved in the computation and the manner in which these values are defined.

\section{Binding attributes and their types}

Assume integer-valued attributes node and Reflndex. 'The attribute node is associated with every node in an S-Structure tree, enumerating them in preorder. 'Thus the node number of an $\mathrm{NP}^{\prime}$ may be used to identify the NP. RefIndex represents the referential index of the NP with which it is associated. This attribute is synthesized by rule (12) and its value is equal to the referential index of the first NP with which the current NP corefers (assuming a preorder enumeration of tree nodes). When NP.RefIndex $=$ NP.node, for some NP, we say the NP has independent reference.

The attribute $A A S$ contains, for a given NP, the sequence of $c$-commanding NPs found within the local domain of the current node. Thus, any NP in this set is a potential antecedent for the current node, if that node is anaphoric. Each element in the $A A S$ is a pair of the form $<N P$. Reflndex, $N{ }^{\prime} . A G R>$, for some NP to the left of the current node. NP's are ordered in the $A A S$ in such way that the most recently found $N P$ is ramked first ( $A A S$ is a stack, or ordered set). The attribute $P A S$ is similar to the $A A S$, except that each element in it

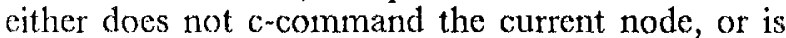
outside its local domain. 'Thus, each NW' in the $P A S$ is a potential antecedent for the current node, if that node is pronominal.

An important difference between the $A A S$ and $P A S$ is that, if the current node is an $N P$, say $N P_{i}$, the pair $\left\langle N P_{i}\right.$.node, $N P_{i} \cdot A G R>$ is a member of $P A S$, but not $\Lambda A S$. Because of this, a pronominal's refcrential index may be set to its own node number (i.c., may be interpreted deictically), while an anaphor's may not. This difference between the $A A S$ and $P A S$ need not be stipulated as a special case, but rather follows naturally if we assume the c-command relation is irreflexive.

The distribution of values for the $A A S$ and $P A S$ attributes in an $\$$-Structure may be illustrated by means of example (13), in which the subscripts are NP node numbers; we ignore their actual values.

(13) John $_{\mathrm{h}}$ told [his ${\text { parents }]_{\mathrm{j}} \text { about himself }}_{\mathrm{k}}$.

The values that result for the $A A S$ and $P A S$ are shown in (14); the reader may verify their correctness with the aid of examples (15). For the first $\mathrm{NP}$, 'John', there is no potential anaphoric antecedent (15.a), so the $A A S$ is empty (14.a). IYowever, at that position it is possible to have a free pronoun, so the $P A S$ contains a single entry, the pair $\left\langle h, A G R_{h}>\right.$. For the second NP, ' $h i s^{\prime}$, the values of $A A S$ and $P A S$ are as in (14.b). Thus the $A A S$ is empty and no anaphor is permissible at the position (15.b), while a pronoun is, in which case it may be interpreted deictically or anaphorically, referring back to 'John'. 'The values of the $A A S$ and $P A S$ attributes associated with $N P$. and $\mathrm{NP}_{\mathrm{k}}$ are as shown in (14.c-d).

$$
\begin{aligned}
& \text { a. } N P_{h} \cdot A A S=\{\} \\
& N D_{h} \cdot P A S=\left\{<h, A G R_{\mathrm{h}_{2}}>\right\} \\
& \text { b. } \mathbf{N P}_{\mathrm{i}}, A A S=\{\} \\
& \left.N P_{i} \cdot P A S=\left\{<i, A G R_{i}\right\rangle,<h, A G R_{h}>\right\} \\
& \text { c. } \mathbb{N P}_{\mathrm{j}}, A A S=\left\{<h, A G R_{\mathrm{h}}>\right\} \\
& \mathbf{N P}_{\mathrm{j}} \cdot P A S=\left\{<j, A G R_{\mathrm{j}}>\right\} \\
& \text { d. } \mathbf{N} \mathbf{P}_{\mathrm{k}} \cdot A A S=\left\{<j, A G R_{\mathrm{j}}>,<h, A G R_{\mathrm{h}}>\right\} \\
& N P_{k} \cdot P A S=\left\{<k, A G R_{\mathrm{k}}>,<i, A G R_{\mathrm{j}}>\right\}
\end{aligned}
$$

(15) a. * Himself $H e_{\mathrm{i}}$ told his parents about himself.
b. John ${ }_{\mathrm{i}}$ told [*himselfs/ his $s_{\mathrm{j} / \mathrm{i}}$ parents] about himself.
c. John told himself $_{\mathrm{i}}$ / "him to stop smoking.
d. John $n_{i}$ told [Mary's parents $]_{k}$ about himself $/$ each other $/$ her $_{\mathrm{h} / \mathrm{j}}$

\footnotetext{
${ }^{2}$ No theoretical significance is attached to the order of the elements in the $A A S$ and $P A S$. Psycholinguistic evidence, however, suggests that gaps "reactivate" their antecedents, which bears on the order of the sets.
} 


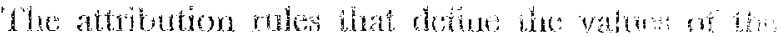

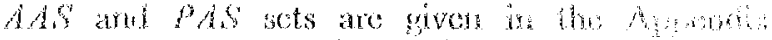

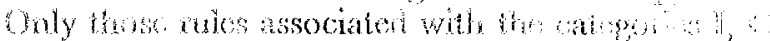

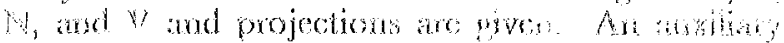

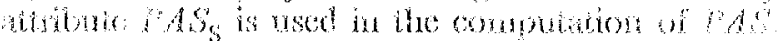

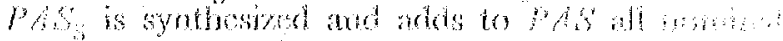

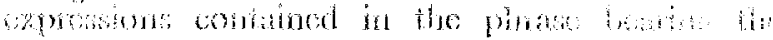
arivbuto.

\section{chuten}

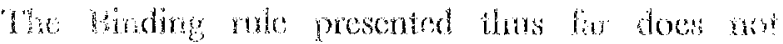

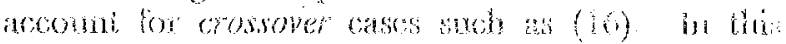
example, Na has undergono Wh-novemoni frons

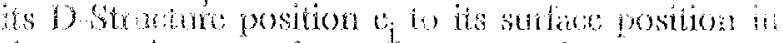

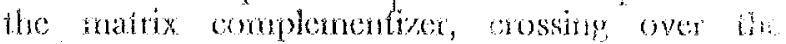

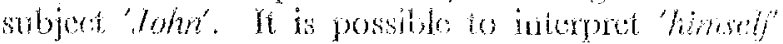

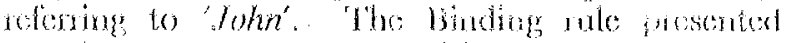
associate; an crapty $A A S$ with himself, and thus fails to arcount for this coreforence possibility.

(10) Which picture of himself $\mathrm{l}_{\mathrm{j}}$ dow foim like $_{\mathrm{j}}$.

Vxample (16) is also problematio fox the axionatio Binding heory defined by (6) (10) since, according io definition (8), the anaphor is uot bound ty its

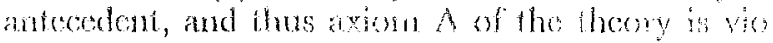

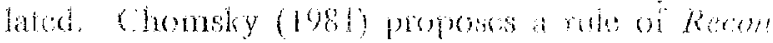

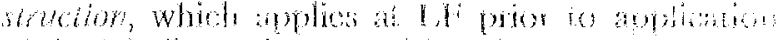

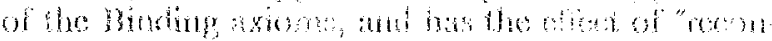

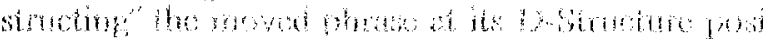

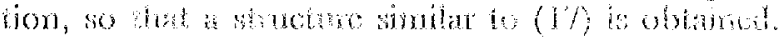

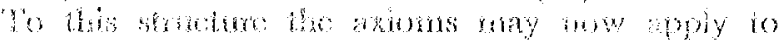

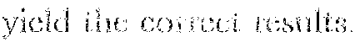

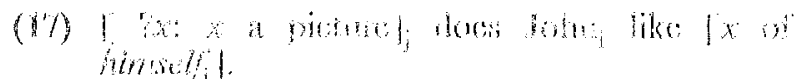

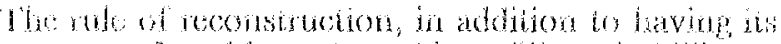
sWr set of problems / van kionadijk and Willans,

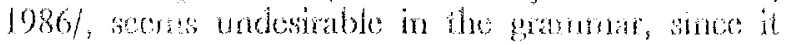
compliceses the gramma spocifoally io the pur poses of the binding theory, 'the function of the

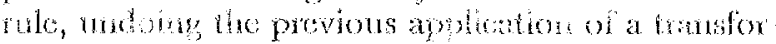
mation, and vory mochling.

Folowng in pat a proposal by $100 \mathrm{se}$ (1983), the

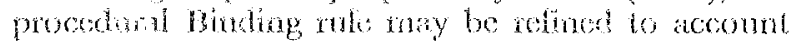
for (16) wibout kooves to reconstruction. Bast observes that the antecedent of an maphor masi

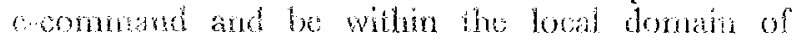
chloe the anapher, of are of the traces of the whese in which the antophor is entedder. This

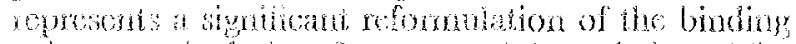

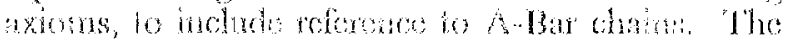

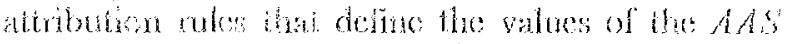

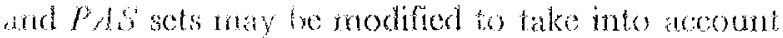

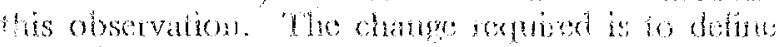
the values at the noot of the novert phese an the

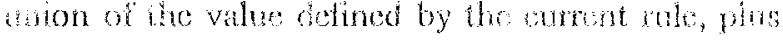

11. Whos dofinct at the traces in the chain beaded , the phase Thus, in (16) NP.AAS and $\mathrm{e}_{\mathrm{j}} A A S$ wate the domon $\mathrm{i}, A G R_{\mathrm{i}}{ }^{3}$, and the attri mon rotes tescribut in the previous section make

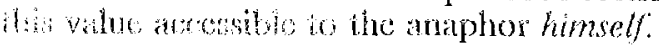

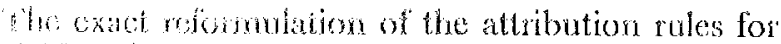

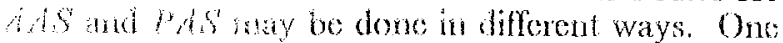
theraive is to esmoute the extended $A A S$ and HA nots in two yessos through the tree, with the weond pasis used to compute the moions noted. $\Lambda$ scoond mperoad delays anaphora resolution for sorphsoms inside at antecedent (chain head) until the was of the chan is fomm. At this tirno ilu dAs and PAS sots of the mecodent may bo svaltatod, having acoss to the comesponding sets it the baces. This second aproach secms more

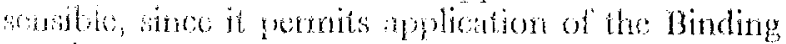
sik: in one loy down, lefi to right pass though the iroe. Wo do not pursic the dotails of the revised rula here.

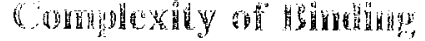

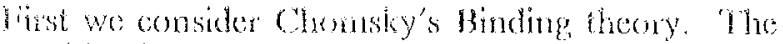
combination of the tree hindexing rule and binding

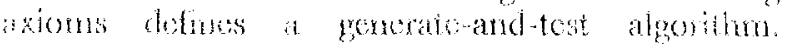

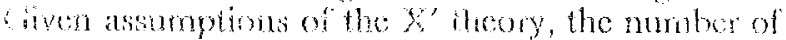

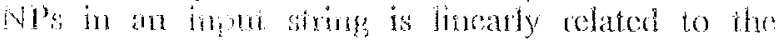

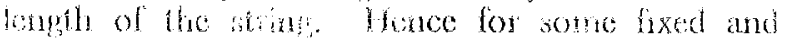

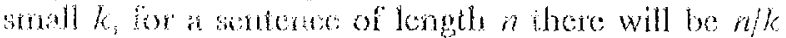
No moles. Assuming a slight modification of the indexing rake (which improves it), according to which if selects intogers in the range $l, \ldots, n / k$ to asign as potontal reforential indices to the Nlys involved, then will bo $(n / k) n / k$ candidate $1 . j$ asignoments to be checked against the Binding axions (1). Assuming that the Bitiding axions way bo checked in constant time, the running tine for the algorithen is exponentially related io the length of the input string.

tion the procedural Binding rule fomulated here, the time meded to compote the synthesized $A A s$ and $P A S$ athibutes at each node from the athibutes at that node on which $A A S$ and $P A S$ directly depend may be assumed to be constant; the operations involved are assignment, push, and pop only. Assuming further that the number of cmoty categories inserted between terninal sements is propotional to the length of the input string, the number of nodes in the derivation trees generated is proportional to the input length. Since the $A A S$ and $P A S$ attribuies are corrouted at most once at cach node in the tres, the processing time for the now Binding rule is lincar a. a significant improvenent over ihe abstract specification (6) (10). 


\section{Jonclusions}

$\mathrm{n}$ this paper an attribute-grammar specification of Binding rule for the identification of pronoun and naphor referents has been proposed. The rule rovides a correct account of backward reference of VP's, and also of forward reference due to movenent, without recourse to reconstruction. The rule resents a model of Binding in which sets of potenial anaphoric and pronominal antecedents are ncrementally defined at each node in a tree. itorage use may be optimized by use of global itorage cells, as described by /Sonnenschein, 1985/.

n more general terms, this rule presents a trend omplementary to that of recent linguistic theory. The rule formulation indicates how conditions on eprescntations may be incorporated into the rules which generate the representations in the first place. This leads to grammars more geared to linguistic rocessing, and to which a higher degree of "psyshological reality" may be ascribed. The rule is a ikcly candidate for incorporation in natural lanzuage parsers.

\section{Acknowledgements}

I would like to thank my dissertation advisors Susumu Kuno and Jaklin Kornfilt for discussion of I3inding, George IJeidorn and Karen Jensen, who read an earlier version of this paper, and Dr. Edward Stabler at Syracuse University for long time support of my dissertation project. Prof. Kuno provided the initial reference to the manuscript by Barss, and kindly made it available.

\section{References}

Abney, Steven, 1986. "Licensing and Parsing." maniuscript, MIT. ${ }^{3}$

Abney, Steven and Jennifer Cole, 1986. "A Government-binding Parser." manuscript, MIT. ${ }^{3}$

Barss, Andrew, 1983. "Chain Binding." manuscript, MIT. ${ }^{3}$.

Barton, Edward, 1984. "Towards a Principledbased Parser." A.I. Memo No. 788, MIT.

Chomsky, Noam, 1981. Lectures on Government and Binding. Foris Publications, Dordrecht.
Chomsky, Noam, 1986. Knowledge of Language. Praeger, New York.

Correa, Nelson, 1987. " $\Lambda$ n Attribute Grammar Implementation of Government-binding Theory." Proceedings of the 25th Annual Meeting of the Association for Computational Linguistics, Stanford, California.

Hobbs, Jerry, 1978. "Resolving Pronoun References." Lingua 44.

Kashket, Michael, 1986. "Parsing a Free-word Order Language." Proceedings of the 24th Annual Meeting of the Association for Computational Linguistics, New York.

Kuhns, Robert, 1986. "A PROLOG Implementation of Government-binding Theory." Proceedings of the 1986 Annual Conference of the European Chapter of the Association for Computational Linguistics,

Kuno, Susumu, 1987. Functional Syntax: Anaphora, Discourse and Empathy. The University of Chicago Press, Chicago, Illinos.

Sharp, Randall, 1985. A Model of Grammar Based on Principles of Government and Binding. M.S. Thesis, Department of Computer Science, University of British Columbia. Vancouver, Canada.

Sells, Peter, 1985. Lectures on Contemporary Linguistic Theories. Chicago University Press, Chicago, Illinois.

Sonnenschein, Michael, 1985. "Global Storage Cells for Attributes in an Attribute Grammar." Acta Informatica 22.

van Riemsdijk, Henk and Edwin Williams, 1986. An Introduction to the Theory of Grammar. 'The MIT Press, Cambridge, Massachussets.

Waite, William M. and Gerhard Goos, 1984. Compiler Construction. Springer-Verlag, New York.

Wehrli, Eric, 1984. "A Government-binding Parser for French." Institut Pour les Etudes Semantiques ef Cognitives, Universite de Geneve. Working Paper No. 48. 
Anperadix: " The Binding rule

(Rules for $A A S$ and PAS computation)

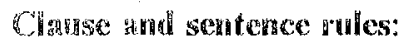

a. $2 \rightarrow \mathbb{C}$

atribution:

CP.PAS $\leftarrow 1]$

CPAS $P[]$

b. $\mathbb{C} P(\mathbb{N} \rightarrow \mathbb{N}) \mathbb{C B}$

atruibution:

CB.AAS - if CP.tense $=+$ then [ ] else CP.AAS

CHP.PAS $\leftarrow$ if CP.tense $=+$

then CP.AAS U CP.PAS else CP.PAS

C.PAS $P A$ setwdiff(CB.PAS $S_{\mathrm{S}}$, CP.AAS)

c. $\mathbb{C H B} \rightarrow \mathbb{C} \mathbb{I P}^{\infty}$

attribution:

$M$ MAS - CH.AAS

HP.PAS CB.PAS

CHB.PAS + IP.PAS

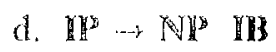

attribution:

NII .AAS

NR.PAS $-[<\mathbb{N P}$.node, NP.AGR $>\mid$ IP.PAS $]$

HA. $A A S$ \& $-[<\mathbb{N P}$. RefIndex, NP.AGR> IP.AAS]

UH.PAS \& IVP.PAS

MP. PAS $A S_{\mathrm{S}}<$

$[<N P$ Reflndex, NP.AGR>| IB.PAS $]$

c. $X$

arribution:

VP.AAS - H.$A A S$

VP.PAS - IMP.PAS

WBP.PAS $A S_{\mathrm{S}}+V P \cdot P A S_{\mathrm{S}}$

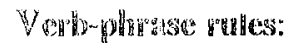

a. $V \mathbb{H}^{3} \rightarrow \ldots W H$

attribution:

VIP.AAS - VP.AAS

VH.PAS VIP.PAS

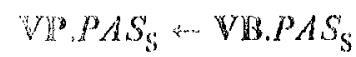

b. $\mathbf{V B} \rightarrow \mathbf{V}$

attribution:

VB.PAS $S_{\mathrm{S}} \leftarrow$ VB. $P A S$

c. $\mathrm{VB} \rightarrow \mathrm{V} X P$, for $\mathrm{XP}=\mathrm{NP}, \mathrm{Cl}$

attribution:

$\mathrm{XP} . A A S \leftarrow \mathrm{VB} . A A S$

$\mathrm{XP} . P A S \leftarrow \mathrm{VB} . P A S$

VB. $P A S_{\mathrm{S}} \leftarrow$ if $\mathrm{XP}=\mathrm{NP}$ then [ $<\mathrm{NP}$.RefIndex, NP.AGR>] else XP.PAS

d. $V B \rightarrow V$ NP $X P$, for $X P=P P, C P$ attribution:

$\mathrm{NP} . A A S \leftarrow \mathrm{VIB} . A A S$

NP.PAS $\leftarrow$ VB.PAS

$\mathrm{XP} . A A S \leftarrow$

$[<\mathrm{Nl}$.Reflndex, NP.AGR>|VIB.AAS]

$\mathrm{XP} . P A S \leftarrow \mathrm{NP} \cdot P A S_{\mathrm{S}}$

VB. $P A S_{\mathrm{S}} \leftarrow \mathrm{XP} . P A S_{\mathrm{S}}$

Noun-phrase rules:

a. $\mathrm{NP} \rightarrow$ (Det) NB

attribution:

$\mathrm{NB} . A A S+\mathrm{NP} . A A S$

$\mathrm{NB} . P A S \leftarrow$ tail $(\mathrm{NP} . P A S)$

NP. $P A S_{\mathrm{S}} \leftarrow \mathrm{NB} \cdot P A S_{\mathrm{S}}$

b. $\mathbf{N P}_{1} \rightarrow \mathbf{N P}_{2} \mathbf{N B}$

attribution:

$\mathrm{NP}_{2} . A A S \leftarrow \mathrm{NP}_{1}, A A S$

$\mathrm{NP}_{2} \cdot P A S$

$\left[<\mathbf{N P}_{2}\right.$.node, $\left.\mathbf{N P}_{2} \cdot A G R>\mid \operatorname{tail}\left(\mathbf{N P}_{1} \cdot P A S\right)\right]$

$\mathrm{NB} . A A S \leftarrow[]$

$\mathrm{NB} . P A S \leftarrow \mathrm{NP}_{2} \cdot P A S_{\mathrm{S}}$

$\mathrm{NP} \mathbb{P}_{1}, P A S_{\mathrm{S}} \leftarrow$

$\left[<\mathrm{NP}_{2} \cdot\right.$ RefIndex, $\left.\mathrm{NP}_{2} \cdot A G R>\mid \mathrm{NP}_{2} \cdot P A S_{\mathrm{S}}\right]$

c. $\mathrm{NB} \rightarrow \mathrm{N}$

attribution:

NB. $P A S_{\mathrm{S}} \leftarrow$ NB. $P A S$

d. $\mathbf{N B} \rightarrow \mathbf{N} X P$, for $\mathbf{X P}=\mathbf{P P}$ or $\mathbf{C P}$

attribution:

$\mathrm{XP} . A A S \leftarrow \mathrm{NB} . A A S$

$\mathrm{XP} . P A S \leftarrow \mathrm{NB} . P A S$

NB. $P A S_{\mathrm{S}} \leftarrow \mathrm{XP} . P A S_{\mathrm{S}}$ 